\title{
Depolarization index from Mueller matrix descatters imaging in turbid water
}

\author{
Fei Liu (刘飞) $)^{1,2,3, \dagger}$, Shichao Zhang (张仕超) ${ }^{1,2, \dagger}$, Pingli Han (韩平丽) $)^{1,2,4}$, Fangyi Chen (陈方熠) ${ }^{1,2}$, Lin Zhao (赵 琳) ${ }^{1,2}$ ， \\ Yingying Fan (范颖颖) $)^{1,2}$, and Xiaopeng Shao (邵晓鹏) $)^{1,2^{*}}$ \\ ${ }^{1}$ School of Physics and Optoelectronic Engineering, Xidian University, Xi'an 710071, China \\ ${ }^{2}$ Xi'an Key Laboratory of Computational Imaging, X'an 710071, China \\ ${ }^{3}$ Academy of Advanced Interdisciplinary Research, Xidian University, X'an 710071, China \\ ${ }^{4}$ State Key Laboratory of Optical Engineering, Chinese Academy of Sciences, Chengdu 610209, China
}

*Corresponding author: xpshao@xidian.edu.cn

Received June 22, 2021 | Accepted August 17, 2021 | Posted Online October 8, 2021

\begin{abstract}
Polarization underwater imaging is of great potential to target detection in turbid water. Typical methods are challenged by the requirement on degrees of polarization (DoPs) of both target light and backscattering. A polarization descattering imaging method was developed using the Mueller matrix, which in turn derived a depolarization (Dep) index from the Mueller matrix to characterize scattering media by estimating the transmittance map by combining a developed optimal function. By quantifying light attenuation with the transmittance map, a clear vision of targets can be recovered. Only using the information of scattering media, the underwater vision under diverse water turbidity was enhanced by the results of experimental data.
\end{abstract}

Keywords: Mueller matrix; scattering; depolarization; underwater imaging.

DOI: 10.3788/COL202220.022601

\section{Introduction}

There have been growing interests in imaging for underwater detection, marine rescue, and aquaculture ${ }^{[1,2]}$. Images taken in such a kind of scattering media are characterized by loss of contrast due to light scattered back from the medium along with the light of sight (backscattering ${ }^{[3-5]}$. Pioneering work towards descattering imaging has been developed including time-gating, laser line scanning, structured lighting, polarization imaging technology, etc. ${ }^{[6-10]}$. Noticeably, polarization imaging technology has become a powerful imaging modality in descattering imaging, benefitting from its simple structure, favorable visualization, and portability ${ }^{[10-12]}$. However, a fundamental issue toward it lies in the requirement of two degrees of polarization (DoPs) of both the object and backscattered light, which is a challenging job. Targets composed of more than one material make DoP calculation complex and further current methods fail. In this paper, the Mueller matrix was employed to develop a polarization descattering approach free from calculating DoP by quantifying how turbid water depolarized light with the parameter depolarization (Dep) index.

The Mueller matrix is mainly exploited and used in biomedical studies benefitting from its distinctive advantages of highcontrast imaging and abundant structure information ${ }^{[13-15]}$.
It works for both linear and circular polarizations ${ }^{[16]}$. Research exploited Mueller matrix imaging for cancer diagnostics, anomalous tissues detection, and so on ${ }^{[17,18]}$. For instance, researchers presented the relationship between the microstructures of different cancerous tissues and the parameters extracted from the Mueller matrix ${ }^{[19]}$. It can also quantify Dep of the optical system.

In this Letter, a polarization descattering imaging method was proposed using a Mueller matrix for clear vision in turbid water. It first exploited the Mueller matrix of waterbody for a Dep, which characterizes how and to what extent the waterbody depolarizes light. Dep, combined with an optimal function, is then used to estimate the transmittance map, which enables quantification of light attenuation in water. The proposed method seeks only scattering information and does not depend on target information and DoP of the medium and target that is needed.

\section{Methods and Results}

According to the Jaffe-McGlamery imaging model ${ }^{[20]}$, a deteriorated underwater image $I_{\text {Total }}(x, y)$ is composed of two parts: the object light $I_{\mathrm{Obj}}(x, y)$ and the backscattered light $I_{\text {Scat }}(x, y)$. An ideal clear image should include only $I_{\text {obj }}(x, y)$ in Eq. (1): 


$$
\begin{aligned}
I_{\text {Obj }}(x, y) & =\frac{I_{\text {Total }}(x, y)-I_{\text {Scat }}(x, y)}{t} \\
& =\frac{I_{\text {Total }}(x, y)-I_{\text {Scat }}^{\infty}(x, y)}{t}+I_{\text {Scat }}^{\infty}(x, y),
\end{aligned}
$$

where $(x, y)$ indicates a pixel location in the image, $t$ represents the medium transmittance map, which quantifies light attenuation along with distance during propagation in water ${ }^{[21]}$, and $I_{\text {Scat }}^{\infty}(x, y)$ is the backscattering intensity at infinity. It is a constant in a determined image and can be calculated by averaging the intensities of the top $0.1 \%$ brightest pixels in $I_{\text {Total }}(x, y)^{[22]}$. Therefore, attention should be paid in the estimation of $t$. The major challenge is to model how it changes at different locations. Though $t$ models attenuation of light intensity, it is challenging to determine it directly by captured intensity images. An approach to estimate $t$ was developed by exploiting polarization, which was an intrinsic characteristic of light. For totally polarized light, it depolarizes during propagation in water due to scattering. This process can be quantified by Dep index, which varies from zero for the total non-depolarizer to one for the ideal depolarizer. Its calculation requires a Mueller matrix of the scattering media, a $4 \times 4$ real-valued matrix describing how media change light polarization ${ }^{[23]}$, as shown by Eq. (2):

$$
\operatorname{Dep}_{M}(x, y)=\frac{\sqrt{\operatorname{tr}\left(M^{T} M\right)-m_{00}^{2}(x, y)}}{\sqrt{3 m_{00}^{2}(x, y)}} .
$$

The Mueller matrix is measured by employing the dual-rotating-retarder method, where two polarizers (LPVISE200-A, Thorlabs) were used, and two achromatic quarter-wave plates (AQWP10M-580, Thorlabs) were mounted on a motorized rotation state (PRM1/MZ8, Thorlabs). Figure 1(a) presents the experiment setup, where illumination is obliquely incident on the target. We dissolved skimmed milk in $100 \mathrm{~L}$ tap water to mimic scattering conditions in turbid water in a $50 \mathrm{~cm} \times$ $80 \mathrm{~cm} \times 40 \mathrm{~cm}$ acrylic tank. The target includes two parts each in different materials, a $15 \mathrm{~cm} \times 2 \mathrm{~cm}$ steel ruler covered by a piece of $3 \mathrm{~cm} \times 2 \mathrm{~cm}$ paper. Four measurements are required to calculate the Mueller matrix. Taken as an example, Fig. 1(b) shows the sixteen images of a Mueller matrix, where the difference between the target and backscattering light is noticeable, as well as between different materials. The Dep index is then calculated by Eq. (2) and shown in Fig. 1(c).

Figures 2(a)-2(d) are the four measured images detected in water with a 30.0 nephelometric turbidity units (NTU), 36.2 NTU, 41.0 NTU, and 50.0 NTU turbidity, respectively. The visibility obviously deteriorated with the turbidity increase. The backscattering intensity is denoted by grayscale values extracted from regions where there is no target. The four colored lines in Fig. 2(e) illustrate backscattering variation. It gets stronger and more uneven with turbidity increasing, and the calculated grayscale values of the Dep index by Eq. (2) are also presented in Fig. 2(e). It also gets stronger under higher turbidity, indicating stronger depolarizing conditions to light. However, Dep is more robust to location than to backscattering, not sensitive to objects and uneven illumination. It is determined only by the medium. Figures $3(\mathrm{~b})-3(\mathrm{e})$ present the distribution of backscattering as a function of Dep in the selected area from images in Fig. 3(a):

$$
I_{\text {Scat }}(x, y)=a \cdot \operatorname{Dep}_{M}(x, y)+b
$$

(a)

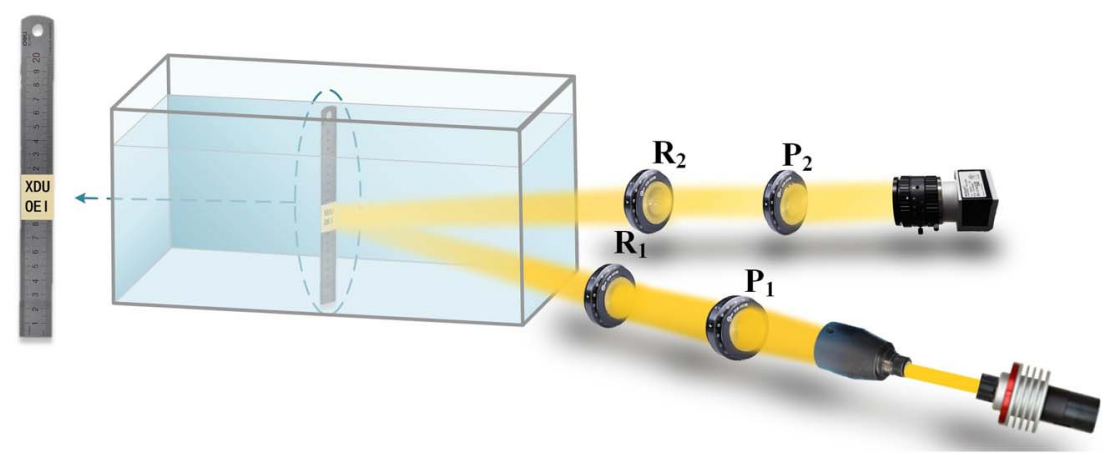

(b)

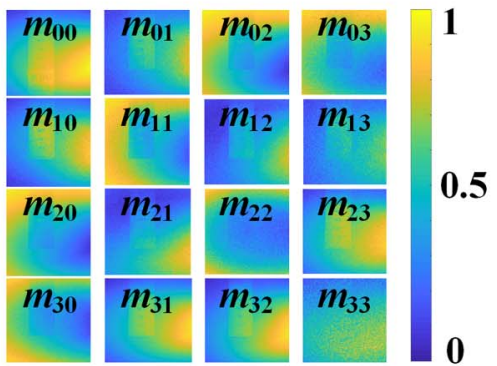

(c)

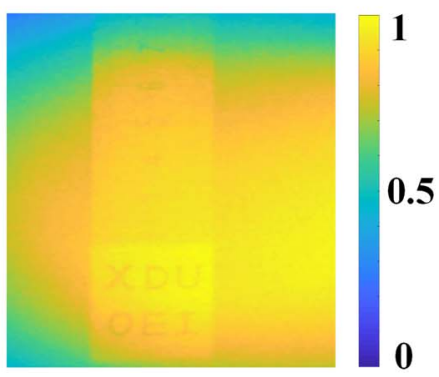

Fig. 1. (a) Experiment setup of the proposed method. P1, P2 are polarizers, and R1, R2 are retarders. (b) and (c) $4 \times 4$ Mueller matrix and Dep index images in the water with a 45 NTU turbidity. 

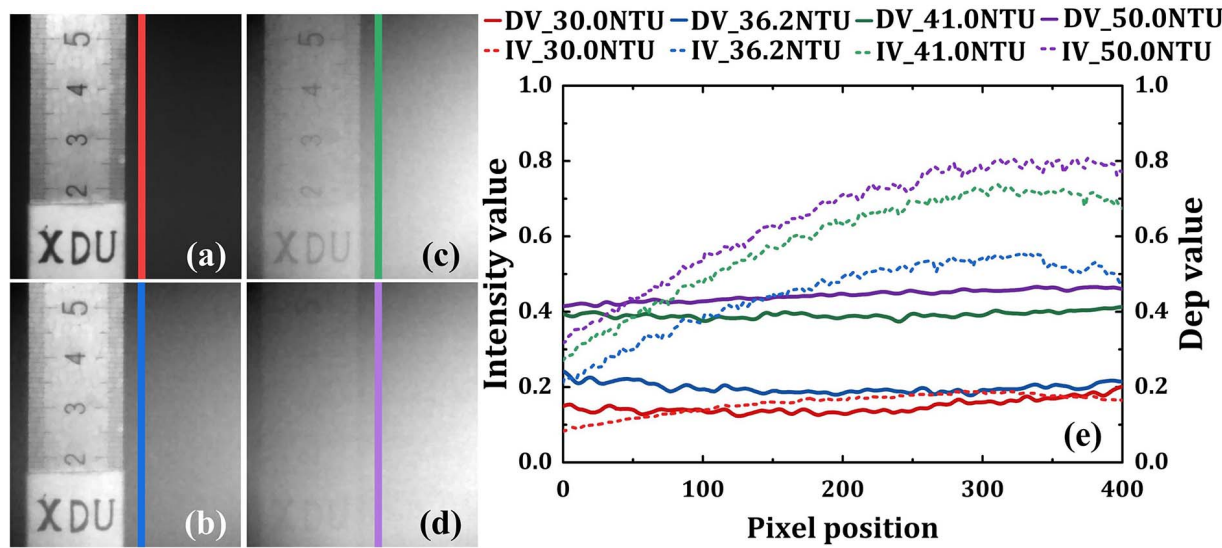

Fig. 2. (a)-(d) Intensity images in the water with 30.0 NTU, 36.2 NTU, 41.0 NTU, and 50.0 NTU turbidity, respectively; (e) intensity and Dep variation of (a)-(d) along the colored lines.
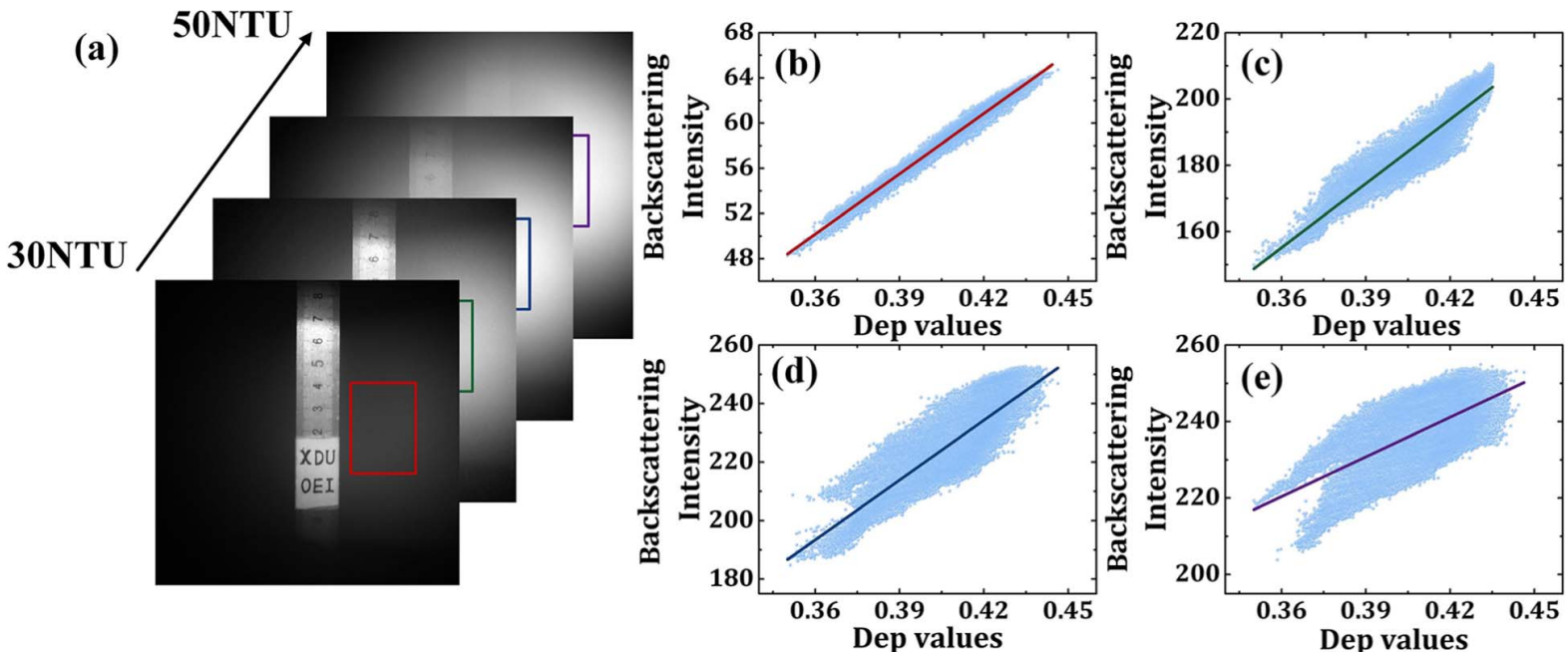

Fig. 3. (a) Four measured $m_{00}(x, y)$ images at 30.0 NTU, 36.2 NTU, 41.0 NTU, and 50.0 NTU; (b)-(e) fitting results of backscattering intensity as a function of Dep values at 30.0 NTU, 36.2 NTU, 41.0 NTU, and 50.0 NTU, respectively.

where $a$ and $b$ are unknown constants. The transmittance map $t$ is determined based upon backscattering, which is directly related to distance. Then, $t$ can be modeled by Dep as Eq. (4) shows:

$$
t=1-\frac{a \cdot \operatorname{Dep}_{M}(x, y)+b}{I_{\text {Scat }}(x, y)}=1-\mathfrak{J} \cdot \operatorname{Dep}_{M}(x, y)-\sigma
$$

where $\sigma$ is an empirical constant, and $\mathfrak{\Im}$ is a parameter varying from 0 to $1 /$ Dep. $\sigma$ only changes the brightness of a recovered image up to a scale and can be neglected in the calculation. Therefore, major attention should be paid to determine $\mathfrak{I}$ during $t$ estimation. Then, the descattering model in Eq. (1) can be optimized into Eq. (5):

$$
I_{\mathrm{Obj}}(x, y)=\frac{m_{00}(x, y)-I_{\mathrm{Scat}}^{\infty}(x, y)}{1-\mathfrak{J} \cdot \operatorname{Dep}_{M}(x, y)-\sigma}+I_{\mathrm{Scat}}^{\infty}(x, y) .
$$

For estimation of $\mathfrak{\Im}$, an underwater image quality measurement (UIQM) ${ }^{[24]}$ was exploited, where two parameters, underwater image sharpness measure (UISM) and underwater image contrast measure (UIConM), were used to evaluate underwater images. Images with higher UIQM are better in image quality. Given the fact that backscattering deteriorates image quality and reduces UISM and UIConM, the optimization is completed by Eq. (6) by searching the optimal $\mathfrak{I}$ enabling the maximum UIQM:

$$
\hat{\mathfrak{I}}=\max _{\mathfrak{I}}\left\{\operatorname{UIQM}\left(I_{\mathrm{Obj}}(x, y), \mathfrak{I}\right)\right\}
$$

where 

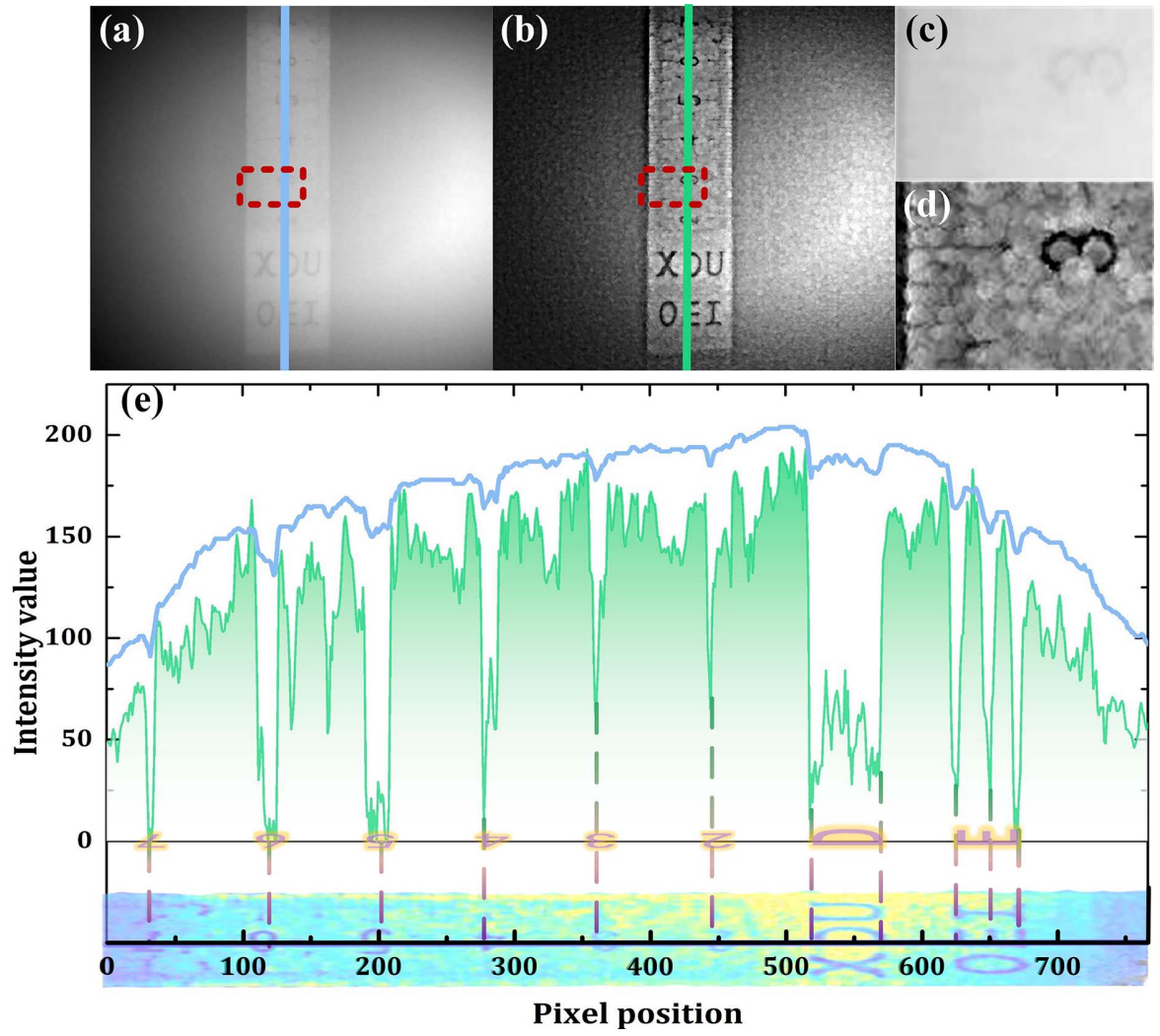

Fig. 4. (a) Intensity image; (b) recovered image with the proposed descattering method; (c) and (d) the zoomed-in view of the region of interest in (a) and (b) marked out with red rectangle; (e) the intensity value variations of (a) and (b) along the colored lines.

$$
\begin{aligned}
\mathrm{UIQM}= & 0.29 \times \mathrm{UISM}+3.57 \times \mathrm{UIConM}, \\
\mathrm{UISM}= & \frac{2}{k_{1} k_{2}} \sum_{l=1}^{k_{1}} \sum_{t=1}^{k_{2}} \log \left(\frac{I_{\max , k, l}}{I_{\min , k, l}}\right), \\
\mathrm{UIConM}= & \frac{1}{k_{1} k_{2}} \otimes \sum_{l=1}^{k_{1}} \sum_{k=1}^{k_{2}} \frac{I_{\max , k, l} \Theta I_{\min , k, l}}{I_{\max , k, l} \bigoplus I_{\min , k, l}} \\
& \times \log \left(\frac{I_{\max , k, l} \Theta I_{\min , k, l}}{I_{\max , k, l} \bigoplus I_{\min , k, l}}\right) .
\end{aligned}
$$

Here, an image is divided into $k_{1} \times k_{2}$ blocks, and $\bigoplus, \otimes$, and $\Theta$ are the parameterized logarithmic image processing operations ${ }^{[24]}$. Finally, with the estimated $\mathfrak{I}$, the clear image can be reconstructed by Eq. (5).

Taking the experiment in 50.0 NTU turbidity water as an example, we show the captured intensity image and the reconstructed image in Figs. 4(a) and 4(b). The image contrast has been greatly improved after recovery. In particular, the paper stripe and metal ruler are simultaneously clearly visible. In the zoomed-in view of the region of interest in Figs. 4(c) and 4(d), the ruler in the intensity image presents a blurred appearance, especially the tick mark and edges. In contrast, after recovery, it is possible to distinguish the exact edges, and even tick marks on the ruler are clearly distinguishable. To quantify clarity improvement, Fig. 4(e) presents extracted details from Figs. 4(a) and $4(\mathrm{~b})$, which is the intensity variation versus the vertical position 521 denoted by the vertical line passing through the Arabic numerals and alphabets. The recovered image provides an obviously intense intensity variation, indicating well-recovered target information and enhanced image contrast where backscattering is well-removed. This benefits from the fact that Dep enables accurate $t(z)$ estimation, since it is robust to location as discussed before. In detail, take the letter " $E$ " as an example. The intensity variation in Fig. 4(e) fits well to its shape. These data preliminarily prove that the method can handle scattering problems in underwater imaging and then improve vision.

Further experiments were conducted under five different turbidities: 30.0 NTU, 36.2 NTU, 41.0 NTU, 45.2 NTU, and 50.0 NTU. Besides the ruler, another set of targets composed of two coins, copper-zinc alloys of 0.5 Chinese Yuan (CNY) and nickel alloys of $1 \mathrm{CNY}$, were tested and shown in Fig. 5. The raw intensity images in the two bottom rows all present an increasing veiling effect with increasing turbidity. However, all of the recovered images keep good vision. For the ruler, clearer details regarding the Arabic numerals as well as enhanced contrast are available after descattering. The two coins as well as their details are clearly visible in the recovered images, where image contrast is also enhanced. In addition, we employed UIQM to quantify image quality before (the dotted line) and after recovery (the full line), as presented by Fig. 5. UIQM works in a similar manner as a human observer, which comprises a sharpness measure (UISM) and a contrast measure (UIConM). It is worth noting that no matter how the water turbidity changes, the 


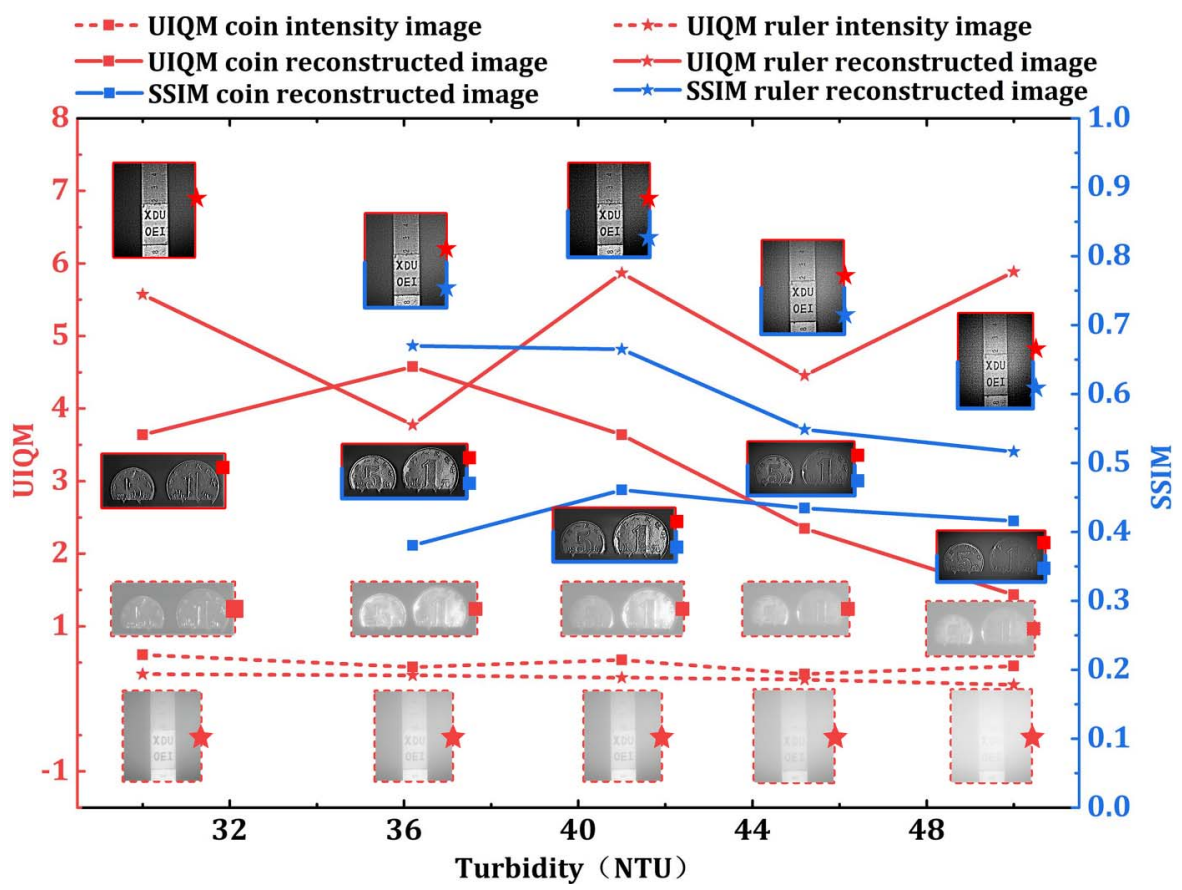

Fig. 5. Experiment results of two sets of targets and evaluation data by UIQM and SSIM.

recovered images keep a significant improvement in image quality. There is a minor fluctuation in the quality of the reconstructed images. This mainly results from the polarization difference caused by water turbidity, even though the overall results are hardly affected. Besides, structural similarity (SSIM) is calculated with the recovered images at 30.0 NTU as reference. The developed method presents robustness to the turbidity increase. Therefore, the developed method is advantageous in robustness to water turbidity.

\section{Conclusion}

In conclusion, we proposed an underwater descattering method using the Mueller matrix to detect targets in multiple materials. It exploited the linear relationship between Dep derived from the Mueller matrix and backscattering intensity. An optimal function was introduced to estimate the transmittance map and then recover clear underwater vision. Experimental results show that the method can assist in detecting targets in multiple materials in turbid underwater. It also may inspire new underwater imaging strategies and has potential to be applied to underwater detection.

\section{Acknowledgement}

This work was supported by the National Natural Science Foundation of China (NSFC) (Nos. 62075175 and 62005203) and the Key Laboratory of Optical Engineering, Chinese Academy of Sciences.

${ }^{\dagger}$ These authors contributed equally to this work.

\section{References}

1. X. Wang, H. Liu, M. Chen, Z. Liu, and S. Han, "Imaging through dynamic scattering media with stitched speckle patterns," Chin. Opt. Lett. 18, 042604 (2020).

2. F. Liu, Y. Wei, P. Han, K. Yang, L. Bai, and X. Shao, "Polarization-based exploration for clear underwater vision in natural illumination," Opt. Express 27, 3629 (2019).

3. P. Han, F. Liu, Y. Wei, and X. Shao, "Optical correlation assists to enhance underwater polarization imaging performance," Opt. Laser Eng. 134, 106256 (2020).

4. S. Yoon, M. Kim, M. Jang, Y. Choi, W. Choi, S. Kang, and W. Choi, "Deep optical imaging within complex scattering media," Nat. Rev. Phys. 2, 141 (2020).

5. K. Liu and Y. Liang, "Underwater image enhancement method based on adaptive attenuation-curve prior," Opt. Express 29, 10321 (2021).

6. M. Wang, X. Wang, L. Sun, Y. Yang, and Y. Zhou, "Underwater 3D deblurring-gated range-intensity correlation imaging," Opt. Lett. 45, 1455 (2020).

7. W. Yi, H. Liu, P. Wang, M. Fu, J. Tan, and X. Li, "Reconstruction of target image from inhomogeneous degradations through backscattering medium images using self-calibration," Opt. Express 25, 7329 (2017).

8. J. Jaffe, "Enhanced extended range underwater imaging via structured illumination," Opt. Express 18, 12328 (2010).

9. J. Jaffe, "Underwater optical imaging: the past, the present, and the prospects," IEEE J. Oceanic Eng. 40, 683 (2014).

10. T. Treibitz and Y. Schechner, "Active polarization descattering," IEEE T. Pattern Anal. 31, 385 (2009).

11. M. Garcia, C. Edmiston, R. Marinov, A. Vail, and V. Gruev, "Bio-inspired color-polarization imager for real-time in situ imaging," Optica 4, 1263 (2017).

12. F. Liu, P. Han, Y. Wei, K. Yang, S. Huang, X. Li, G. Zhang, L. Bai, and X. Shao, "Deeply seeing through highly turbid water by active polarization imaging," Opt. Lett. 43, 4903 (2018).

13. M. Kudenov, M. Escuti, N. Hagen, E. Dereniak, and K. Oka, "Snapshot imaging Mueller matrix polarimeter using polarization gratings," Opt. Lett. 37, 1367 (2012).

14. A. Tariq, H. He, P. Li, and H. Ma, "Purity-depolarization relations and the components of purity of a Mueller matrix," Opt. Express 27, 22645 (2019). 
15. K. Yan, S. Wang, S. Jiang, L. Xue, Y. Song, Z. Yan, and Z. Li, "Calculation and analysis of Mueller matrix in light scattering detection," Chin. Opt. Lett. 12, 092901 (2014).

16. G. Kattawar and D. Gray, "Mueller matrix imaging of targets in turbid media: effect of the volume scattering function," Appl. Opt. 42, 7225 (2003).

17. A. Pierangelo, A. Benali, M. Antoneli, T. Novikova, P. Novikova, P. Validire, B. Gayet, and A. Martino, "Ex-vivo characterization of human colon cancer by Mueller polarimetric imaging," Opt. Express 19, 1582 (2011).

18. Y. Dong, J. Qi, H. He, C. He, S. Liu, J. Wu, D. S. Elson, and H. Ma, "Quantitatively characterizing the microstructural features of breast ductal carcinoma tissues in different progression stages by Mueller matrix microscope," Biomed. Opt. Express 8, 3643 (2017).

19. C. He, H. He, J. Chang, Y. Dong, S. Liu, N. Zeng, Y. He, and H. Ma, "Characterizing microstructures of cancerous tissues using multispectral transformed Mueller matrix polarization parameters," Biomed. Opt. Express 6, 2934 (2015)

20. J. Jaffe, "Computer modeling and the design of optimal underwater imaging systems,” IEEE J. Oceanic Eng. 15, 101 (1990).

21. T. Treibitz and Y. Schechner, "Active polarization descattering," IEEE T. Pattern Anal. 3, 385 (2008).

22. K. Amer, M. Elbouz, A. Alfalou, C. Brosseau, and J. Hajjami, "Enhancing underwater optical imaging by using a low-pass polarization filter," Opt. Express 27, 621 (2019).

23. R. Chipman, "Depolarization index and the average degree of polarization," Appl. Opt. 44, 2490 (2005).

24. K. Panetta, C. Gao, and S. Agaian, "Human-visual-system-inspired underwater image quality measures,” IEEE J. Oceanic Eng. 41, 541 (2016). 